\title{
Pacific
}

Journal of

Mathematics

\section{ASYMPTOTICALLY FREE FAMILIES OF RANDOM UNITARIES IN SYMMETRIC GROUPS}

AleXANDRU Mihai NiCA 


\title{
ASYMPTOTICALLY FREE FAMILIES OF RANDOM UNITARIES IN SYMMETRIC GROUPS
}

\author{
AleXANDRU Nica
}

\begin{abstract}
We prove that independent, Haar distributed families of random unitaries in symmetric groups are asymptotically free.
\end{abstract}

1. Introduction. In this note we prove that independent, Haar distributed families of random unitaries in symmetric groups are asymptotically free.

If $G_{n}$ is a closed subgroup of the unitary group $U(n)$, then by a random unitary in $G_{n}$ we understand a measurable function $f: X \rightarrow G_{n}$, where $(X, \mathscr{F}, P)$ is a (fixed) probability space. A random unitary in $G_{n}$ has a distribution, which is a probability measure on $G_{n}$, and we can define the notion of independence for a family of random unitaries, exactly as it is done for usual real-valued random variables. A family $\left(f_{\omega}\right)_{\omega \in \Omega}$ of random unitaries in $G_{n}$ will be called, following [1], standard-independent if it is independent and if the distribution of every $f_{\omega}$ is the Haar measure on $G_{n}$.

Now, a family $\left(f_{\omega}\right)_{\omega \in \Omega}$ of random unitaries in $G_{n}$ can be also viewed as a family of unitaries in the non-commutative probability space $\left(\mathfrak{M}_{n}, \tau_{n}\right)$, where $\mathfrak{M}_{n}$ is the unital *-algebra of measurable functions $X \rightarrow \operatorname{Mat}_{n}(\mathbb{C})$, having bounded entries, and $\tau_{n}$ is the tracestate of $\mathfrak{M}_{n}$ obtained by integrating the normalized trace of $\operatorname{Mat}_{n}(\mathbb{C})$. From this point of view, the concept analogous to independence to be considered is the property of $\left(f_{\omega}\right)_{\omega \in \Omega}$ of being or not being free (see [2]). This property can be expressed in terms of a naturally defined "non-commutative distribution" of $\left(f_{\omega}\right)_{\omega \in \Omega}$, which is a state on the group algebra of the free group on $\Omega$ generators.

Hence, we are in a situation when both concepts of independence and freeness can be considered. It seems to be a deep phenomenon that, as found by D. Voiculescu in [1] for several important examples of such situations, one can hope independence to give rise (at least in good cases) to asymptotic freeness.

In our particular framework, the problem-type reflecting this phenomenon can be stated as follows: "For every $n \geq 1$, let $\left(f_{n, \omega}\right)_{\omega \in \Omega}$ 
be a standard-independent family of random unitaries in the closed subgroup $G_{n}$ of $U(n)$. Is it true, for a reasonable series $\left(G_{n}\right)_{n=1}^{\infty}$, that the families $\left(f_{n}, \omega\right)_{\omega \in \Omega}$ are asymptotically free for $n \rightarrow \infty$ ?" In [1] it is proved that this is true for $G_{n}=U(n)$, and it is found that the limit non-commutative distribution of the families $\left(f_{n, \omega}\right)_{\omega \in \Omega}$ is, roughly speaking, the free product of $\Omega$ copies of the Haar measure on the circle.

The goal of this paper is to prove that the same holds if every $G_{n}$ is a semidirect product $A_{n}^{n} \times{ }_{\alpha_{n}} S_{n}$, with $S_{n}$ the group of permutations of $\{1,2, \ldots, n\}, A_{n}$ a closed subgroup of the circle, and $\alpha_{n}: S_{n} \rightarrow$ $\operatorname{Aut}\left(A_{n}^{n}\right)$ the natural action,

$$
\alpha_{n}(t)\left(z_{1}, z_{2}, \ldots, z_{n}\right)=\left(z_{t^{-1}(1)}, z_{t^{-1}(2)}, \ldots, z_{t^{-1}(n)}\right) .
$$

The paper is subdivided into sections as follows: in $\S 2$ we fix the notations and review the concepts related to non-commutative probability spaces that we need. In $\S 3$ we state precisely the problem-type concerning asymptotic freeness for standard-independent families of random unitaries. In $\S 4$ we prove the main theorem of the paper, namely that asymptotic freeness holds for the series $\left(S_{n}\right)_{n=1}^{\infty}$ of symmetric groups, and in $\S 5$ we extend the result to the above-mentioned case of semidirect products.

We are deeply indebted to Dan Voiculescu for his constant support during the preparation of this work. We are thankful for the good atmosphere of the Operator Algebra theme year at the C. R. M., Montreal, where the paper was written.

2. Basic definitions. In this section we fix the notations and briefly review the basic concepts about non-commutative random variables that we need (for a more detailed exposition, see [2]).

2.1. By a non-commutative probability space we shall understand a pair $(\mathfrak{A}, \sigma)$, with $\mathfrak{A}$ a unital *-algebra and $\sigma$ a state of $\mathfrak{A}$ (i.e. $\sigma: \mathfrak{A} \rightarrow \mathbb{C}$ linear, $\sigma\left(a^{*} a\right) \geq 0$ for any $\left.a \in \mathfrak{A}, \sigma(1)=1\right)$.

2.2. ${ }^{*}$-distributions for families of unitaries. For a non-void set $\Omega$, let $F(\Omega)$ be the free group having a free family of generators indexed by $\Omega$, and let $\mathbb{C}[F(\Omega)]$ be its group algebra. It is handy to view $\mathbb{C}[F(\Omega)]$ as an algebra of non-commutative trigonometric polynomials, i.e., as having a linear basis consisting of 1 and the monomials $X_{\omega_{1}}^{\alpha_{1}} X_{\omega_{2}}^{\alpha_{2}} \cdots X_{\omega_{m}}^{\alpha_{m}}$, with $\omega_{1} \neq \omega_{2} \neq \cdots \neq \omega_{m}$ in $\Omega$ and $\alpha_{1}, \alpha_{2}, \ldots, \alpha_{m}$ in $\mathbb{Z} \backslash\{0\}$. (Note: By $\omega_{1} \neq \omega_{2} \neq \cdots \neq \omega_{m}$ we shall always mean that $\omega_{i} \neq \omega_{i+1}, 1 \leq i \leq m-1$.) Of course, if card $\Omega=1$, then $\mathbb{C}[F(\Omega)]=\mathbb{C}[\mathbb{Z}]$ is the algebra of usual trigonometric polynomials. 
$\mathbb{C}[F(\Omega)]$ has a natural *-operation, uniquely determined by the condition that every $X_{\omega}(\omega \in \Omega)$ be unitary, and has the following universality property: for any unital *-algebra $\mathfrak{A}$, and for any family $\left(u_{\omega}\right)_{\omega \in \Omega}$ of unitaries of $\mathfrak{A}$, there exists a unique homomorphism of unital *-algebras, $\Pi: \mathbb{C}[F(\Omega)] \rightarrow \mathfrak{A}$, such that $\Pi\left(X_{\omega}\right)=u_{\omega}$, for every $\omega$ in $\Omega$.

Definition. Let $(\mathfrak{A}, \sigma)$ be a non-commutative probability space. The *-distribution of a unitary $u \in \mathfrak{A}$ will be the state $\mu_{u}: \mathbb{C}[\mathbb{Z}] \rightarrow \mathbb{C}$ obtained by composing $\sigma$ with the unique homomorphism $\mathbb{C}[\mathbb{Z}] \rightarrow \mathfrak{A}$ sending $X$ into $u$. More generally, the *-distribution of a family $\left(u_{\omega}\right)_{\omega \in \Omega}$ of unitaries in $\mathfrak{A}$ will be the state $\mu: \mathbb{C}[F(\Omega)] \rightarrow \mathbb{C}$ obtained by composing $\sigma$ with the unique homomorphism $\mathbb{C}[F(\Omega)] \rightarrow \mathfrak{A}$ sending $X_{\omega}$ into $u_{\omega}$ for every $\omega$.

2.3. Example (Haar distribution on $\mathbb{C}[\mathbb{Z}]$ ). Consider the algebra $C(\mathbb{T})$ of continuous functions on the circle, with pointwise operations, let $\sigma: C(\mathbb{T}) \rightarrow \mathbb{C}$ be integration with respect to Haar measure, and let id $\in C(\mathbb{T})$ be the unitary id $(z)=z$. The *-distribution $\mu_{\text {id }}: \mathbb{C}[\mathbb{Z}] \rightarrow \mathbb{C}$ will be called the Haar distribution on $\mathbb{C}[\mathbb{Z}]$; it is clearly determined by the property that $\mu_{\mathrm{id}}\left(X^{k}\right)=0$ for any $k \neq 0$.

2.4. Free families. Let $(\mathfrak{A}, \sigma)$ be a non-commutative probability space. A family $\left(u_{\omega}\right)_{\omega \in \Omega}$ of unitaries in $\mathfrak{A}$ is called free if for any $\omega_{1} \neq \omega_{2} \neq \cdots \neq \omega_{m}$ in $\Omega$ and $p_{1}, p_{2}, \ldots, p_{m}$ in $\mathbb{C}[\mathbb{Z}]$ such that $\sigma\left(p_{j}\left(u_{\omega_{j}}\right)\right)=0,1 \leq j \leq m$, we also have that $\sigma\left(p_{1}\left(u_{\omega_{1}}\right) \cdots p_{m}\left(u_{\omega_{m}}\right)\right)$ $=0$.

Remark that the fact whether the family $\left(u_{\omega}\right)_{\omega \in \Omega}$ is free or not depends only on its *-distribution $\mu$ on $\mathbb{C}[F(\Omega)]$. More precisely, it is easy to check that $\left(u_{\omega}\right)_{\omega \in \Omega}$ is free in $(\mathfrak{A}, \sigma)$ if and only if $\left(X_{\omega}\right)_{\omega \in \Omega}$ is free in $(\mathbb{C}[F(\Omega)], \mu)$.

2.5. Asymptotically free families. Let, for every $n \geq 1,\left(u_{n}, \omega\right)_{\omega \in \Omega}$ be a family of unitaries in the non-commutative probability space $\left(\mathfrak{A}_{n}, \sigma_{n}\right)$, and let $\mu_{n}$ be its *-distribution. The families $\left(u_{n, \omega}\right)_{\omega \in \Omega}$ are said to converge in distribution (for $n \rightarrow \infty$ ) to the state $\mu$ on $\mathbb{C}[F(\Omega)]$ if $\mu_{n}(p) \underset{n \rightarrow \infty}{\rightarrow} \mu(p)$, for any $p$ in $\mathbb{C}[F(\Omega)]$. If, moreover, the limit state $\mu$ has the property that the family $\left(X_{\omega}\right)_{\omega \in \Omega}$ is free in $(\mathbb{C}[F(\Omega)], \mu)$, then the families $\left(u_{n, \omega}\right)_{\omega \in \Omega}$ are said to be asymptotically free for $n \rightarrow \infty$.

2.6. Random matrices. For $(X, \mathscr{F}, P)$ a probability space and $n$ a positive integer, we shall work with the unital *-algebra $\mathfrak{M}_{n}$ of 
measurable functions $f: X \rightarrow \operatorname{Mat}_{n}(\mathbb{C})$ having the property that for any $1 \leq i, j \leq n$, the entry $f_{i, j}: X \rightarrow \mathbb{C}$ is bounded. This is only a subalgebra of what one usually calls the algebra of $n \times n$ random matrices on $(X, \mathscr{F}, P)$ (see the definitions preceding Theorem 2.2 of [1]), but it will be sufficient for our purposes. On $\mathfrak{M}_{n}$ we have a canonical trace state, defined by:

$$
\tau_{n}(f)=\frac{1}{n} \int_{X} \operatorname{Tr} f(x) d P(x)=\frac{1}{n} \sum_{j=1}^{n} \int_{X} f_{j, j} d P .
$$

2.7. Random unitaries. Let $(X, \mathscr{F}, P)$ and $n$ be as above, and assume that we are also given a closed subgroup $G$ of $U(n)$. A measurable function $f: X \rightarrow G$ (which is in particular a unitary in the algebra $\mathfrak{M}_{n}$ defined at 2.6) will be called a random unitary in $G$.

As a unitary in the non-commutative probability space $\left(\mathfrak{M}_{n}, \tau_{n}\right)$, a random unitary $f$ in $G$ has of course a ${ }^{*}$-distribution in the sense of 2.2. But in this case, we also have the distribution of $f$ defined in the classical sense, which is the probability measure on the Borel $\sigma$-algebra of $G$, given by the formula: $\lambda_{f}(A)=P\left(f^{-1}(A)\right)$. More generally, a finite family $\left(f_{\omega}\right)_{\omega \in \Omega}$ of random unitaries in $G$ has a joint distribution, which is the probability measure $\lambda$ on the compact group $G^{\Omega}$, given by

$$
\lambda(A)=P\left(\left\{x \in X \mid\left(f_{\omega}(x)\right)_{\omega \in \Omega} \in A\right\}\right), \quad A \subseteq G^{\Omega} \text { Borel set } .
$$

If $\lambda=\prod_{\omega \in \Omega} \lambda_{f_{\omega}}$, the family $\left(f_{\omega}\right)_{\omega \in \Omega}$ is called independent. If this happens and, moreover, each $\lambda_{f_{\omega}}$ coincides with the Haar measure on $G$, the family $\left(f_{\omega}\right)_{\omega \in \Omega}$ will be called standard-independent (see 3.7 of [1]).

An arbitrary family (finite or not) $\left(f_{\omega}\right)_{\omega \in \Omega}$ of random unitaries in $G$ will be called standard-independent if so is $\left(f_{\omega}\right)_{\omega \in \Omega_{0}}$ for any finite subset $\Omega_{0}$ of $\Omega$. As it is easily checked, this is equivalent to the fact that

$$
\int_{X} \varphi_{1}\left(f_{\omega_{1}}(x)\right) \cdots \varphi_{m}\left(f_{\omega_{m}}(x)\right) d P(x)=\prod_{q=1}^{m} \int_{G} \varphi_{q}(t) d t
$$

for any $\omega_{1}, \ldots, \omega_{m}$ in $\Omega$ such that $\omega_{i} \neq \omega_{j}$ when $i \neq j$, and for any $\varphi_{1}, \ldots, \varphi_{m}$ in $C(G)$; on the right side of (2), integration is done with respect to the Haar measure of $G$. 


\section{The setting of the problem.}

3.1. The enunciation of the problem can be done as follows. For every $n \geq 1$, let $\left(f_{n}, \omega\right)_{\omega \in \Omega}$ be a standard-independent family of random unitaries in the closed subgroup $G_{n}$ of $U(n)$. Is it true, for reasonable series $\left(G_{n}\right)_{n=1}^{\infty}$, that:

(i) the families $\left(f_{n, \omega}\right)_{\omega \in \Omega}$, regarded in the non-commutative probability spaces $\left(\mathfrak{M}_{n}, \tau_{n}\right)$, are asymptotically free, and

(ii) For every $\omega \in \Omega$, the unitaries $f_{n, \omega}$ converge in distribution (for $n \rightarrow \infty$ ) to the Haar distribution on $\mathbb{C}[\mathbb{Z}]$ ?

3.2. Reformulation. For concrete computations, it is useful to remark that (i) and (ii) of 3.1 together are equivalent to the following assertion: For any $\omega_{1} \neq \omega_{2} \neq \cdots \neq \omega_{m}$ in $\Omega$, and any $\alpha_{1}, \alpha_{2}, \ldots, \alpha_{m}$ in $\mathbb{Z} \backslash\{0\}$, we have:

$$
\lim _{n \rightarrow \infty} \tau_{n}\left(f_{n, \omega_{1}}^{\alpha_{1}} \cdots f_{n, \omega_{m}}^{\alpha_{m}}\right)=0
$$

Indeed, (i) + (ii) mean that the ${ }^{*}$-distributions of the families $\left(f_{n, \omega}\right)_{\omega \in \Omega}$ converge, for $n \rightarrow \infty$, to a state $\mu$ on $\mathbb{C}[F(\Omega)]$ with the following properties:

(j) $\left(X_{\omega}\right)_{\omega \in \Omega}$ is a free family in $(\mathbb{C}[F(\Omega)], \mu)$;

(jj) $\mu\left(X_{\omega}^{k}\right)=0$ for any $\omega$ in $\Omega$ and $k$ in $\mathbb{Z} \backslash\{0\}$.

But (j) and (jj) together are clearly equivalent to

(jjj) $\mu\left(X_{\omega_{1}}^{\alpha_{1}} \cdots X_{\omega_{m}}^{\alpha_{m}}\right)=0$, for any $\omega_{1} \neq \omega_{2} \neq \cdots \neq \omega_{m}$ in $\Omega$ and $\alpha_{1}, \alpha_{2}, \ldots, \alpha_{m}$ in $\mathbb{Z} \backslash\{0\}$

(in particular $(\mathrm{j})+(\mathrm{jj})$ determine $\mu$ completely).

So, if (i) and (ii) hold, we have for any $\omega_{1} \neq \omega_{2} \neq \cdots \neq \omega_{m}$ in $\Omega$ and any $\alpha_{1}, \ldots, \alpha_{m}$ in $\mathbb{Z} \backslash\{0\}$ :

$$
\lim _{n \rightarrow \infty} \tau_{n}\left(f_{n, \omega_{1}}^{\alpha_{1}} \cdots f_{n, \omega_{m}}^{\alpha_{m}}\right)=\mu\left(X_{\omega_{1}}^{\alpha_{1}} \cdots X_{\omega_{m}}^{\alpha_{m}}\right)=0
$$

conversely, if (3) holds, then the *-distributions of the families $\left(f_{n, \omega}\right)_{\omega \in \Omega}$ converge for $n \rightarrow \infty$ to a state on $\mathbb{C}[F(\Omega)]$ satisfying (jjj), and we have (i) + (ii).

3.3. REMARK. The expression $\tau_{n}\left(f_{n, \omega_{1}}^{\alpha_{1}} \cdots f_{n, \omega_{m}}^{\alpha_{m}}\right)$ appearing in (3) depends in fact only on $G_{n}$ (and not on the probability space $(X, \mathscr{F}, P)$ we started with). Indeed, if $f_{n, \omega_{1}}^{\alpha_{1}} \cdots f_{n, \omega_{m}}^{\alpha_{m}}=f$, then for any $1 \leq j \leq n$, the entry $f_{j, j}$ of $f$ is a polynomial in the entries of $f_{n, \omega_{1}}, \ldots, f_{n, \omega_{m}}$, and $\int_{X} f_{j, j} d P$ is seen not to depend on $(X, \mathscr{F}, P)$ because of (2) of 2.7 .

3.4. Reformulation for $G$ finite. Let us assume that the subgroups $G_{n} \subseteq U(n)$ considered at 3.1 are finite. 
Let $\left(f_{n, \omega}\right)_{\omega \in \Omega}$ be a family of random unitaries in $G_{n}$. Since $C\left(G_{n}\right)$ is the linear span of characteristic functions of one-point sets, and Haar measure on $G_{n}$ is the normalized counting measure, the relation (2) of 2.7 (i.e. the standard-independence of $\left.\left(f_{n, \omega}\right)_{\omega \in \Omega}\right)$ is seen to be equivalent to

$$
P\left(f_{n, \omega_{1}}^{-1}\left(t_{1}\right) \cap \cdots \cap f_{n, \omega_{m}}^{-1}\left(t_{m}\right)\right)=\frac{1}{\left(\operatorname{card} G_{n}\right)^{m}},
$$

for any $\omega_{1}, \ldots, \omega_{m}$ in $\Omega$ such that $\omega_{i} \neq \omega_{j}$ when $i \neq j$, and for any $t_{1}, \ldots, t_{m}$ in $G_{n}$.

Further, let $\left(f_{n, \omega}\right)_{\omega \in \Omega}$ be a standard-independent family of random unitaries in $G_{n}$, and let us compute $\tau_{n}\left(f_{n, \omega_{1}}^{\alpha_{1}} \cdots f_{n, \omega_{m}}^{\alpha_{m}}\right)$ for some $\omega_{1} \neq \omega_{2} \neq \cdots \neq \omega_{m}$ in $\Omega$ and $\alpha_{1}, \ldots, \alpha_{m}$ in $\mathbb{Z} \backslash\{0\}$. When $q$ runs from 1 to $m, \omega_{q}$ describes a subset $\omega_{1}^{\prime}, \ldots, \omega_{k}^{\prime}$ of $\Omega$, with $k \leq m$. In other words, we have written $\omega_{q}=\omega_{c_{q}}^{\prime},(1 \leq q \leq m)$, with $\omega_{i}^{\prime} \neq \omega_{j}^{\prime}$ for $i \neq j$. The hypothesis $\omega_{1} \neq \omega_{2} \neq \cdots \neq \omega_{m}$ becomes $c_{1} \neq c_{2} \neq \cdots \neq c_{m}$.

We claim that

$$
\tau_{n}\left(f_{n, \omega_{1}}^{\alpha_{1}} \cdots f_{n, \omega_{m}}^{\alpha_{m}}\right)=\frac{1}{n\left(\operatorname{card} G_{n}\right)^{k}} \sum_{t_{1}, \ldots, t_{k} \in G_{n}} \operatorname{Tr}\left(t_{c_{1}}^{\alpha_{1}} \cdots t_{c_{m}}^{\alpha_{m}}\right) .
$$

Indeed, for any $t_{1}, \ldots, t_{k}$ in $G_{n}$, the function $f_{n, \omega_{1}}^{\alpha_{1}} \cdots f_{n, \omega_{m}}^{\alpha_{m}}$ is constant and equal to $t_{c_{1}}^{\alpha_{1}} \cdots t_{c_{m}}^{\alpha_{m}}$ on the set $f_{n, \omega_{1}^{\prime}}^{-1}\left(t_{1}\right) \cap \cdots \cap f_{n, \omega_{k}^{\prime}}^{-1}\left(t_{k}\right)$, which has measure $1 /\left(\operatorname{card} G_{n}\right)^{k}$, by (4). The sets of this form realise a partition of $X$ (when $t_{1}, \ldots, t_{k}$ describe $G_{n}$ ); decomposing the integral which appears in the formula (1) of 2.6 after this partition, we get (5).

We conclude that a sufficient condition for having an affirmative answer to (i) and (ii) of 3.1 (for any indexing set $\Omega$ ) is, in this case

$$
\lim _{n \rightarrow \infty} \frac{1}{n\left(\operatorname{card} G_{n}\right)^{k}} \sum_{t_{1}, \ldots, t_{k} \in G_{n}} \operatorname{Tr}\left(t_{c_{1}}^{\alpha_{1}} \cdots t_{c_{m}}^{\alpha_{m}}\right)=0
$$

for any $k \geq 1, c_{1} \neq c_{2} \neq \cdots \neq c_{m}$ exhausting $\{1, \ldots, k\}$ and $\alpha_{1}, \ldots, \alpha_{m}$ in $\mathbb{Z} \backslash\{0\}$. Clearly, this condition is also necessary (take $\Omega=\{1, \ldots, k\}$ and $\left.\omega_{1}=c_{1}, \ldots, \omega_{m}=c_{m}\right)$.

\section{Asymptotic freeness in the case of the symmetric groups.}

4.1. Statement of the result. We view the symmetric group $S_{n}$ as a subgroup of $U(n)$, by identifying every $t$ in $S_{n}$ with the corresponding permutation matrix (the entry $(i, j)$ equals 1 if $t(j)=i$, and 0 otherwise). We shall prove that: 
THEOREM. The assertions (i) and (ii) of 3.1 are true for the series $\left(S_{n}\right)_{n=1}^{\infty}$.

As we saw at 3.4 , this comes to

$$
\lim _{n \rightarrow \infty} \frac{1}{n(n !)^{k}} \sum_{t_{1}, \ldots, t_{k} \in S_{n}} \operatorname{Tr}\left(t_{c_{1}}^{\alpha_{1}} \cdots t_{c_{m}}^{\alpha_{m}}\right)=0,
$$

for any $k \geq 1, c_{1} \neq c_{2} \neq \cdots \neq c_{m}$ exhausting $\{1, \ldots, k\}$ and $\alpha_{1}, \ldots, \alpha_{m}$ in $\mathbb{Z} \backslash\{0\}$.

The numbers: $k, c_{1}, \ldots, c_{m}, \alpha_{1}, \ldots, \alpha_{m}$ will be fixed for the rest of this section. In fact, we shall also fix for the rest of the section an integer $n$, not too small (for instance such that $n>$ $\left.2\left(\left|\alpha_{1}\right|+\cdots+\left|\alpha_{m}\right|\right)\right)$, and prove the inequality:

$$
\frac{1}{n(n !)^{k}} \sum_{t_{1}, \ldots, t_{k} \in S_{n}} \operatorname{Tr}\left(t_{c_{1}}^{\alpha_{1}} \cdots t_{c_{m}}^{\alpha_{m}}\right) \leq \frac{1}{n}(\alpha+2)^{\alpha},
$$

with $\alpha=\left|\alpha_{1}\right|+\cdots+\left|\alpha_{m}\right|$. Clearly, (8) implies (7) and hence the proposition.

4.2. REMARK. In some particular cases, the left side of (8) can be computed precisely. We give here some examples (we omit the proofs, since they are not part of the main stream of this paper).

$1^{\circ}$. Assume that there exists $1 \leq j \leq k$ with the following property: there is only one $q(1 \leq q \leq m)$ with $c_{q}=j$, and for that $q$ we have $\alpha_{q}= \pm 1$. Then the left side of $(8)$ is exactly $1 / n$.

$2^{\circ}$. Assume that for every $1 \leq j \leq k$ there is only one $q(1 \leq q \leq$ $m$ ) with $c_{q}=j$ (but instead there is no condition on the exponents). After a change of indices, the non-commutative monomial $t_{c_{1}}^{\alpha_{1}} \cdots t_{c_{m}}^{\alpha_{m}}$ becomes $t_{1}^{\alpha_{1}} \cdots t_{k}^{\alpha_{k}}$. We have

$$
\frac{1}{n(n !)^{k}} \sum_{t_{1}, \ldots, t_{k} \in S_{n}} \operatorname{Tr}\left(t_{1}^{\alpha_{1}} \cdots t_{k}^{\alpha_{k}}\right)=\frac{1}{n}+\frac{\prod_{j=1}^{k}\left(\Delta\left(\alpha_{j}\right)-1\right)}{n(n-1)^{k-1}}
$$

where $\Delta\left(\alpha_{j}\right)$ denotes the number of positive divisors of $\alpha_{j}$.

$3^{\circ}$. Assume that the non-commutative monomial $t_{c_{1}}^{\alpha_{1}} \cdots t_{c_{m}}^{\alpha_{m}}$ is the commutator of two permutations. We have:

$$
\frac{1}{n(n !)^{2}} \sum_{t_{1}, t_{2} \in S_{n}} \operatorname{Tr}\left(t_{1} t_{2} t_{1}^{-1} t_{2}^{-2}\right)=\frac{1}{n-1} .
$$

It would be interesting to find such precise evaluations in the general case. 
4.3. Notations. (a) Besides $k, c_{1}, \ldots, c_{m}, \alpha_{1}, \ldots, \alpha_{m}$ and $\alpha=\left|\alpha_{1}\right|+\cdots+\left|\alpha_{m}\right|$ which were fixed at 4.1 , we also fix the following partition of $\{1,2, \ldots, \alpha\}$ into integer valued intervals:

$$
\begin{aligned}
& I_{1}=\left\{1, \ldots,\left|\alpha_{1}\right|\right\} \text {, } \\
& I_{2}=\left\{\left|\alpha_{1}\right|+1, \ldots,\left|\alpha_{1}\right|+\left|\alpha_{2}\right|\right\} \text {, } \\
& I_{m}=\left\{\left|\alpha_{1}\right|+\cdots+\left|\alpha_{m-1}\right|+1, \ldots,\left|\alpha_{1}\right|+\cdots+\left|\alpha_{m-1}\right|+\left|\alpha_{m}\right|\right\} .
\end{aligned}
$$

(b) Relations. By a relation we shall mean a subset of $\{1, \ldots, n\}^{2}$. A relation $R$ will be called injective if the two projections on the components are injective when restricted to $R$. For any $t$ in $S_{n}$, the relation $R_{t}$ associated to $t$ will be $\{(i, j) \mid t(i)=j\}$.

It is easy to see that for a given relation $R$, there exist permutations $t$ such that $R \subseteq R_{t}$ if and only if $R$ is injective; if this happens, the number of permutations $t$ such that $R \subseteq R_{t}$ equals $(n-(\operatorname{card} R))$ !.

(c) Cycles. By a cycle we shall understand a sequence $\xi=\left(u_{1}, \ldots\right.$, $\left.u_{\alpha}, u_{\alpha+1}\right)$ of numbers in $\{1, \ldots, n\}$, such that $u_{\alpha+1}=u_{1}$. To a cycle $\xi$ we shall associate $k$ relations, $R_{1}(\xi), \ldots, R_{k}(\xi)$. It will be useful at 4.7 to have the construction made for any sequence $\eta=\left(u_{1}, \ldots, u_{\beta}\right)$ of elements of $\{1, \ldots, n\}$, with $2 \leq \beta \leq \alpha+1$. So, having such a sequence $\eta$, we define for any $1 \leq j \leq k$ a relation $R_{j}(\eta)$ as follows: we take all the numbers $1 \leq a \leq \beta-1$ which belong to intervals $I_{q}$ $\left(1 \leq q \leq m\right.$ ) having $c_{q}=j$; and for any such $a \in I_{q}$ we take into $R_{j}(\eta)$ the couple:

$$
\begin{cases}\left(u_{a+1}, u_{a}\right), & \text { if } \alpha_{q}>0 \\ \left(u_{a}, u_{a+1}\right), & \text { if } \alpha_{q}<0\end{cases}
$$

A sequence $\eta=\left(u_{1}, \ldots, u_{\beta}\right)$ will be called injective if the relations $R_{1}(\eta), \ldots, R_{k}(\eta)$ are so.

Having a cycle $\xi$ and a $k$-tuple $\left(t_{1}, \ldots, t_{k}\right)$ in $S_{n}^{k}$, we shall write $\xi \prec\left(t_{1}, \ldots, t_{k}\right)$ if $R_{j}(\xi) \subseteq R_{t_{j}}$, for all $1 \leq j \leq k$.

Now, for $\xi=\left(u_{1}, \ldots, u_{\alpha}, u_{\alpha+1}\right)$ a cycle, $t$ a permutation and $1 \leq j \leq k$, we clearly have: $R_{j}(\xi) \subseteq R_{t} \Leftrightarrow t^{\operatorname{sign} \alpha_{q}}\left(u_{a+1}\right)=u_{a}$, for any $1 \leq q \leq m$ such that $c_{q}=j$, and for any $a \in I_{q}$. This gives the criterion

$$
\begin{aligned}
\xi=\left(u_{1}, \ldots, u_{\alpha}, u_{\alpha+1}\right) \prec\left(t_{1}, \ldots, t_{k}\right) \\
\Leftrightarrow t_{c_{q}}^{\operatorname{sign} \alpha_{q}}\left(u_{a+1}\right)=u_{a}, \quad \forall 1 \leq q \leq m, a \in I_{q} .
\end{aligned}
$$


4.4. Lemma. For any $t_{1}, \ldots, t_{k}$ in $S_{n}$ we have:

$$
\operatorname{Tr}\left(t_{c_{1}}^{\alpha_{1}} \cdots t_{c_{m}}^{\alpha_{m}}\right)=\operatorname{card}\left\{\xi \operatorname{cycle} \mid \xi \prec\left(t_{1}, \ldots, t_{k}\right)\right\} .
$$

Proof. Let $t_{c_{q}} \stackrel{\text { sign } \alpha_{q}}{\text { def }}=s_{q}=\left(s_{q ; u, v}\right)_{1 \leq u, v \leq n} \in U(n)$. Then:

$$
\begin{aligned}
\operatorname{Tr}\left(t_{c_{1}}^{\alpha_{1}} \cdots t_{c_{m}}^{\alpha_{m}}\right) & =\operatorname{Tr}\left(s_{1}^{\left|\alpha_{1}\right|} \cdots s_{m}^{\left|\alpha_{m}\right|}\right) \\
& =\sum_{\xi=\left(u_{1}, \ldots, u_{\alpha+1}\right), \text { cycle }}\left(\prod_{q=1}^{m} \prod_{a \in I_{q}} s_{q ; u_{a}, u_{a+1}}\right) .
\end{aligned}
$$

Since $s_{q ; u, v}$ equals 1 if $t_{c_{q}}^{\operatorname{sign} \alpha_{q}}(v)=u$, and 0 otherwise, every term of the last sum is 0 or 1 , and it is 1 if and only if $t_{c_{q}}^{\operatorname{sign} \alpha_{q}}\left(u_{a+1}\right)=u_{a}$ for every $1 \leq q \leq m$ and $a \in I_{q}$. Comparing with (9) we obtain the desired equality.

4.5. Lemma. For any injective cycle $\xi$ we have

$$
\begin{aligned}
& \operatorname{card}\left\{\left(t_{1}, \ldots, t_{k}\right) \in S_{n}^{k} \mid \xi \prec\left(t_{1}, \ldots, t_{k}\right)\right\} \\
& \quad \leq(n !)^{k}(n / 2)^{-\sum_{j=1}^{k} \operatorname{card} R_{j}(\xi)} .
\end{aligned}
$$

Proof. We have:

$$
\left\{\left(t_{1}, \ldots, t_{k}\right) \in S_{n}^{k} \mid \xi \prec\left(t_{1}, \ldots, t_{k}\right)\right\}=\prod_{j=1}^{k}\left\{t \in S_{n} \mid R_{t} \supseteq R_{j}(\xi)\right\},
$$

so that the cardinal to be majorized is

$$
\begin{aligned}
\prod_{j=1}^{k} \operatorname{card}\left\{t \in S_{n} \mid R_{t} \supseteq R_{j}(\xi)\right\} \\
\left.=\prod_{j=1}^{k}\left(n-\left(\operatorname{card} R_{j}(\xi)\right)\right) ! \quad(\text { by } 4.3 \mathrm{~b})\right) \\
=(n !)^{k} n^{-\sum_{j=1}^{k} \operatorname{card} R_{j}(\xi)} \\
\quad \cdot \prod_{j=1}^{k}\left(\frac{n}{n} \cdot \frac{n}{n-1} \cdots \cdots \cdot \frac{n}{n-\left(\operatorname{card} R_{j}(\xi)\right)+1}\right) .
\end{aligned}
$$

Now, from $4.3 \mathrm{c}$ ) it is clear that $\sum_{j=1}^{k} \operatorname{card} R_{j}(\xi) \leq \sum_{q=1}^{m} \operatorname{card} I_{q}=$ $\alpha$. This implies that $\frac{n}{n}, \frac{n}{n-1}, \ldots, \frac{n}{n-(\operatorname{card} R(\xi))+1}$ are not greater than $\frac{n}{n-\alpha+1}<2$ (we assumed in 4.1 that $n$ is not too small); majorizing all these factors with 2 in the last expression, we get (10). 
4.6. A reduction of the problem. Applying Lemma 4.4, changing the order of summation and applying after that Lemma 4.5 we get

$$
\begin{aligned}
& \frac{1}{n(n !)^{k}} \sum_{t_{1}, \ldots, t_{k} \in S_{n}} \operatorname{Tr}\left(t_{c_{1}}^{\alpha_{1}} \cdots t_{c_{m}}^{\alpha_{m}}\right) \\
& =\frac{1}{n(n !)^{k}} \sum_{t_{1}, \ldots, t_{k} \in S_{n}} \operatorname{card}\left\{\xi \operatorname{cycle} \mid \xi \prec\left(t_{1}, \ldots, t_{k}\right)\right\} \\
& \quad=\frac{1}{n(n !)^{k}} \sum_{\xi \text { cycle }} \operatorname{card}\left\{\left(t_{1}, \ldots, t_{k}\right) \in S_{n}^{k} \mid \xi \prec\left(t_{1}, \ldots, t_{k}\right)\right\} \\
& \leq \frac{1}{n(n !)^{k}} \sum_{\xi \text { injective cycle }}(n !)^{k}(n / 2)^{-\sum_{\jmath=1}^{k} \operatorname{card} R_{J}(\xi)} .
\end{aligned}
$$

The sum $\sum_{j=1}^{k}$ card $R_{j}(\xi)$ takes values not greater than $\alpha$, as remarked in the proof of 4.5 , and not less than $k$ (since obviously every $R_{j}(\xi)$ is non-void). Hence the last expression equals:

$$
\frac{1}{n} \sum_{l=k}^{\alpha}(2 / n)^{l} \text { card }\left\{\xi \text { injective cycle } \mid \sum_{j=1}^{k} \operatorname{card} R_{j}(\xi)=l\right\},
$$

and a simple computation shows that (8) will follow if we can prove that:

$$
\operatorname{card}\left\{\xi \mid \xi \text { injective cycle, } \sum_{j=1}^{k} \operatorname{card} R_{j}(\xi)=l\right\} \leq\left(\begin{array}{c}
\alpha \\
l
\end{array}\right) \alpha^{\alpha-l} n^{l}
$$

for every $k \leq l \leq \alpha$.

4.7. The tree of injective cycles. In order to estimate the cardinals of sets of injective cycles needed in (11), it is convenient to have all the injective cycles placed together in a rooted tree.

Let $T$ be a rooted tree, let $V$ be its set of vertices, and let $v_{0}$ be its root. For any $v$ in $V$, the length of the (unique) path connecting $v$ and $v_{0}$ will be called the level of $v$, and denoted by $L(v)$. The vertices of level $L(v)+1$ which can be connected with $v$ by a path of length 1 will be called the successors of $v$; their number, denoted by $D(v)$, will be called the degree of $v$.

The rooted tree $T$ will be called $(1, n)$-regular if

(i) it has a maximal value of the levels, $L_{\max } \geq 2$;

(ii) any vertex $v$ with $L(v) \neq L_{\max }$ has $D(v)=1$ or $n-\alpha \leq$ $D(v) \leq n$ (of course, for $L(v)=L_{\max }$ we have $D(v)=0$ ). 
Clearly, if $T$ is a $(1, n)$-regular rooted tree, then for any vertex $v$ of $T$, the subtree $T_{v}$ of $T$ generated by $v$ is also $(1, n)$-regular.

By a labeling of the $(1, n)$-regular tree $T$ we shall understand a function $e: V \backslash\left\{v_{0}\right\} \rightarrow\{1, \ldots, n\}$, having the property that whenever $v \in V$ has $n-\alpha \leq D(v) \leq n$, the restriction of $e$ to the set of the successors of $v$ is one-to-one.

Proposition. One can construct a rooted $(1, n)$-regular tree $T$, with $L_{\max }=\alpha+1$, and a labeling $e: V \backslash\left\{v_{0}\right\} \rightarrow\{1, \ldots, n\}$ such that

$1^{\circ}$. For any $2 \leq \beta \leq \alpha+1$, there is a canonical bijection from $\{v \in V \mid L(v)=\beta\}$ onto the injective sequences of $\beta$ numbers in $\{1, \ldots, n\}$ (see 4.3c)), given by the following rule: for any $v$ in $V$ with $L(v)=\beta$, we take the unique path $v_{0}, v_{1}, \ldots, v_{\beta}=v$ connecting $v$ to the root, and we associate to it the sequence $\left(e\left(v_{1}\right), \ldots, e\left(v_{\beta}\right)\right)$.

$2^{\circ}$. For any $v$ in $V$ with $n-\alpha \leq D(v) \leq n$ and $L(v) \leq \alpha-1$, at most $\alpha$ of the successors of $v$ have degree 1 .

$3^{\circ}$. Let $\xi$ be an injective cycle, an let $v$ be the unique vertex with $L(v)=\alpha+1$ associated to $\xi$ at $1^{\circ}$. Then, denoting the path between $v_{0}$ and $v$ by $v_{0}, v_{1}, \ldots, v_{\alpha+1}=v$, we have:

$$
\operatorname{card}\left\{1 \leq \beta \leq \alpha \mid D\left(v_{\beta}\right)=1\right\}=\alpha-\sum_{j=1}^{k} \operatorname{card} R_{j}(\xi) .
$$

Proof. We shall construct the levels of the tree inductively, and define the labeling at the same time, taking care that $1^{\circ}$ holds.

The level 0 contains only one vertex, the root, which is not labeled. The level 1 contains $n$ vertices, labeled from 1 to $n$. The level 2 contains $n^{2}$ vertices, and more precisely, every vertex of the level 1 has $n$ successors, labeled from 1 to $n$. It is clear that the rule described at $1^{\circ}$ gives a bijection between the vertices of the level 2 and the sequences of two numbers in $\{1, \ldots, n\}$ (which are all injective). If $\alpha=1$, then this is $T$, and $1^{\circ}, 2^{\circ}, 3^{\circ}$ are easily checked. For the rest of the proof, we shall suppose that $\alpha \geq 2$.

Now, let us assume that for some $2 \leq \beta \leq \alpha$ we have constructed the tree and the labeling up to the level $\beta$, such that $1^{\circ}$ is satisfied. For constructing the level $\beta+1$, what we have to do is provide an algorithm which decides, for a given vertex $v$ with $L(v)=\beta$, what $D(v)$ should be, and which indicates the labels of the successors of $v$. Let $q(1 \leq q \leq m)$ be such that $\beta \in I_{q}$, and consider $j=c_{q} \in$ $\{1, \ldots, k\}, \alpha_{q} \in \mathbb{Z} \backslash\{0\}$. If $\alpha_{q}>0$, the algorithm sounds like this: 
"Take $v$ with $L(v)=\beta$, consider the path $v_{0}, v_{1}, \ldots, v_{\beta}=v$ connecting $v$ to the root, consider the sequence $\eta=\left(e\left(v_{1}\right), \ldots, e\left(v_{\beta}\right)\right)$ and the relation $R_{j}(\eta)$ (defined at 4.3c)). If there is $u \in\{1, \ldots, n\}$ such that $\left(u, e\left(v_{\beta}\right)\right) \in R_{j}(\eta)$, then put $D(v)=1$, and the label of the unique successor of $v$ is $u$. Otherwise, $n-\alpha \leq D(v) \leq n$, and the labels of the successors of $v$ are $\{1, \ldots, n\} \backslash \pi_{1}\left(R_{j}(\eta)\right) . "$ If $\alpha_{q}<0$, the algorithm is the same, but we replace " $\left(u, e\left(v_{\beta}\right)\right)$ " with " $\left(e\left(v_{\beta}\right), u\right)$ ".

It is easy to check that the level $\beta+1$ constructed in this manner has the property of $1^{\circ}$. Hence the construction can be reiterated up to $\beta=\alpha$, giving us a labeled $(1, n)$-regular rooted tree $T$, with maximal value of the levels $\alpha+1$, and satisfying $1^{\circ}$.

To prove $2^{\circ}$, we need the following

LEMMA. Let $v^{\prime}$ be in $V$ such that $L\left(v^{\prime}\right)=\beta^{\prime} \leq \alpha$ and $D\left(v^{\prime}\right)=1$. Let $v_{0}, v_{1}, \ldots, v_{\beta^{\prime}}=v^{\prime}$ be the path connecting $v^{\prime}$ with the root. Then there exists $1 \leq \gamma \leq \beta^{\prime}-1$ such that $e\left(v^{\prime}\right)=e\left(v_{\gamma}\right)$.

Proof of the Lemma. We denote by $q, q^{\prime}$, respectively, the numbers in $\{1, \ldots, m\}$ such that $\beta^{\prime}-1 \in I_{q}, \beta^{\prime} \in I_{q^{\prime}}$ (note that $L\left(v^{\prime}\right)=\beta^{\prime}$, $D\left(v^{\prime}\right)=1$ imply $\beta^{\prime} \geq 3$, so that $q$ makes sense; clearly, $q=q^{\prime}$ or $\left.q=q^{\prime}-1\right)$.

Let us assume that $e\left(v^{\prime}\right) \neq e\left(v_{\gamma}\right)$ for every $1 \leq \gamma \leq \beta^{\prime}-1$, and obtain a contradiction. To make a choice, suppose that $\alpha_{q^{\prime}}>0$. From $D\left(v^{\prime}\right)=1$ and the way we constructed the tree, we infer that there exists $1 \leq \gamma \leq \beta^{\prime}-1$, belonging to an interval $I_{p}$, such that $c_{p}=c_{q^{\prime}}$ and:

$$
\begin{cases}e\left(v_{\gamma}\right)=e\left(v^{\prime}\right), & \text { if } \alpha_{p}>0, \\ e\left(v_{\gamma+1}\right)=e\left(v^{\prime}\right), & \text { if } \alpha_{p}<0 .\end{cases}
$$

Because of the assumptions we made, the only possibility is that $\alpha_{p}<$ 0 and $\gamma=\beta^{\prime}-1$ (hence $p=q$ ). This gives $c_{q}=c_{q^{\prime}}$ and $\alpha_{q}<0$. Further, $\alpha_{q}<0<\alpha_{q^{\prime}}$ implies $q \neq q^{\prime}$, and hence $q=q^{\prime}-1$; so we get $c_{q}=c_{q-1}$, a contradiction. If $\alpha_{q^{\prime}}<0$, we proceed in the same manner.

The proof of $2^{\circ}$ is now immediate. Take $v$ in $V$ with $L(v)=$ $\beta \leq \alpha-1, n-\alpha \leq D(v) \leq n$, and let $v_{0}, v_{1}, \ldots, v_{\beta}=v$ be the unique path connecting $v$ with the root. If a successor $v^{\prime}$ of $v$ has $D\left(v^{\prime}\right)=1$, then, by the lemma, $e\left(v^{\prime}\right) \in\left\{e\left(v_{1}\right), \ldots, e\left(v_{\beta}\right)\right\}$ which has at most $\alpha-1$ elements. Since the labeling is one-to-one on the set of successors of $v$, we obtain $2^{\circ}$. 
Finally, let $\xi, v$ and $v_{0}, v_{1}, \ldots, v_{\alpha+1}=v$ be as in $3^{\circ}$. From the construction of the tree it is clear that, for any $2 \leq \beta \leq \alpha$ :

$$
\begin{aligned}
& \sum_{j=1}^{k} \operatorname{card} R_{j}\left(e\left(v_{1}\right), \ldots, e\left(v_{\beta}\right), e\left(v_{\beta+1}\right)\right) \\
& \quad-\sum_{j=1}^{k} \operatorname{card} R_{j}\left(e\left(v_{1}\right), \ldots, e\left(v_{\beta}\right)\right)= \begin{cases}0, & \text { if } D\left(v_{\beta}\right)=1 ; \\
1, & \text { if } n-\alpha \leq D\left(v_{\beta}\right) \leq n .\end{cases}
\end{aligned}
$$

Hence the sum:

$\operatorname{card}\left\{1 \leq \gamma \leq \beta \mid D\left(v_{\gamma}\right)=1\right\}+\sum_{j=1}^{k} \operatorname{card} R_{j}\left(e\left(v_{1}\right), \ldots, e\left(v_{\beta}\right), e\left(v_{\beta+1}\right)\right)$,

considered for $1 \leq \beta \leq \alpha$, increases with 1 when $\beta$ increases with 1 . Since for $\beta=1$ :

$$
\operatorname{card}\left\{1 \leq \gamma \leq 1 \mid D\left(v_{\gamma}\right)=1\right\}+\sum_{j=1}^{k} \operatorname{card} R_{j}\left(e\left(v_{1}\right), e\left(v_{2}\right)\right)=0+1=1,
$$

by putting $\beta=\alpha$ we get assertion $3^{\circ}$.

4.8. Remarks on $(1, n)$-regular rooted trees.

4.8.1. Lemma. Let $T$ be a rooted tree with maximal value of the levels $L_{\max }=\beta+1$, and such that every vertex $v$ with $L(v) \leq \beta$ has $D(v) \leq n$. Then, for any $0 \leq \gamma \leq \beta+1, T$ has at most $n^{\gamma}$ vertices of level $\gamma$.

The proof of 4.8.1 is clear, by induction on $\gamma$.

4.8.2. Lemma. Let $T$ be a $(1, n)$-regular rooted tree, with maximal value of the levels $\beta+1$, and with the following property: for any vertex $v$ with $L(v) \leq \beta-1$ and $n-\alpha \leq D(v) \leq n$, at most $\alpha$ of the successors of $v$ have degree 1 . Then for any $1 \leq \gamma \leq \beta$, there are no more than $\alpha n^{\gamma-1}$ vertices of degree 1 on the level $\gamma$.

Proof. The case $\gamma=1$ is clear. If $\gamma \geq 2$, denote by $N_{\delta}^{(i)}$ the number of vertices of degree $i$ on the level $\delta, i \in\{1, \ldots, n\}, \delta \in\{\gamma-1, \gamma\}$. We have

$$
N_{\gamma}^{(1)} \leq \alpha \sum_{i=n-\alpha}^{n} N_{\gamma-1}^{(i)}+N_{\gamma-1}^{(1)} \leq \alpha \sum_{i=1}^{n} N_{\gamma-1}^{(i)} \stackrel{4.8 .1}{\leq} \alpha n^{\gamma-1} .
$$


4.8.3. LEMMA. Let $T$ be $a(1, n)$-regular rooted tree with the property stated at 4.8.2. Fix $1 \leq \beta_{1} \leq \cdots \leq \beta_{h} \leq \beta$, and let $N$ be the number of vertices with $L(v)=\beta$ and with the following property: if $v_{0}, v_{1}, \ldots, v_{\beta}=v$ is the path connecting $v$ with the root, then $D\left(v_{\beta_{1}}\right)=\cdots=D\left(v_{\beta_{h}}\right)=1$. Then $N \leq \alpha^{h} n^{\beta-h}$.

Proof. We first take the case $h=1$. Let $V_{\beta_{1}}^{(1)}$ be the set of vertices of degree 1 on the level $\beta_{1}$, and for any $v$ in $V_{\beta_{1}}^{(1)}$, let $T_{v}$ be the subtree of $T$ generated by $v$. Clearly, $N=\sum_{v \in V_{\beta_{1}}^{(1)}} N_{v}$, with $N_{v}$ the number of vertices of $T_{v}$ having level $\beta-\beta_{1}$ (in $T_{v}$ ). 4.8.1 gives us that $N_{v} \leq n^{\beta-\beta_{1}} \quad\left(v \in V_{\beta_{1}}^{(1)}\right)$, and 4.8.2 that $\operatorname{card} V_{\beta_{1}}^{(1)} \leq \alpha n^{\beta_{1}-1}$; hence $N \leq \alpha n^{\beta-1}$.

We now make induction on $h$. Assume the lemma proved for all the possible choices $1 \leq \beta_{1}<\cdots<\beta_{h} \leq \beta \quad$ ( $\beta$ natural) and let us prove it for a system $1 \leq \beta_{1}<\cdots<\beta_{h}<\beta_{h+1} \leq \beta$. Defining $V_{\beta_{1}}^{(1)}$ as in the preceding paragraph, we have again the formula $N=\sum_{v \in V_{\beta_{1}}^{(1)}} N_{v}$, where this time $N_{v}$ is the number to be majorized with respect to the tree $T_{v}$ and the system $1 \leq \beta_{2}-\beta_{1}<\cdots<\beta_{h+1}-\beta_{1} \leq \beta-\beta_{1}$. Hence, by 4.8.2 and the induction hypothesis:

$$
N \leq\left(\alpha n^{\beta_{1}-1}\right)\left(\alpha^{h} n^{\beta-\beta_{1}-h}\right)=\alpha^{h+1} n^{\beta-(h+1)} .
$$

4.8.4. Proposition. Let $T$ be a $(1, n)$-regular rooted tree, with the property stated at 4.8.2, and with the maximal value of the levels $\alpha+1$. Let $0 \leq h \leq \alpha$ be a fixed integer. For any vertex $v$ with $L(v)=\alpha$, we consider the path $v_{0}, v_{1}, \ldots, v_{\alpha}=v$ connecting $v$ to the root. Then

$$
\operatorname{card}\left\{v \mid L(v)=\alpha, \operatorname{card}\left\{1 \leq \beta \leq \alpha \mid D\left(v_{\beta}\right)=1\right\} \geq h\right\} \leq\left(\begin{array}{l}
\alpha \\
h
\end{array}\right) \alpha^{h} n^{\alpha-h} .
$$

Proof. For $h=0$ we have to prove that the number of vertices of level $\alpha$ is not greater than $n^{\alpha}$ (which is in 4.8.1). If $h \geq 1$, then for any $v$ having $\operatorname{card}\left\{1 \leq \beta \leq \alpha \mid D\left(v_{\beta}\right)=1\right\} \geq h$ we choose a system $1 \leq \beta_{1}<\cdots<\beta_{h} \leq \alpha$ such that $D\left(v_{\beta_{1}}\right)=\cdots=D\left(v_{\beta_{h}}\right)=1$; after that, we sum after all the possible choices of $1 \leq \beta_{1}<\cdots<\beta_{h} \leq \alpha$, and apply 4.8.3.

4.9. End of the proof of Theorem 4.1. We were left to prove (11) of 4.6. Let us fix $l(k \leq l \leq \alpha)$, and denote $\alpha-l$ by $h$. We consider 
the tree $T$ of the injective sequences, constructed at 4.7. To every injective cycle $\xi$ having $\sum_{j=1}^{k}$ card $R_{j}(\xi)=l$ we associate the unique vertex $v$ of this tree such that: $L(v)=\alpha$, and $\left(e\left(v_{1}\right), \ldots, e\left(v_{\alpha}\right)\right)$ are the first $\alpha$ components of $\xi$ (as usual, $v_{0}, v_{1}, \ldots, v_{\alpha}=v$ is the path connecting $v$ to the root). Taking into account point $3^{\circ}$ of Proposition 4.7, we see that $\xi \rightarrow v$ is a one-to-one mapping into the set of vertices $\left\{v \mid L(v)=\alpha, \operatorname{card}\left\{1 \leq \beta \leq \alpha \mid D\left(v_{\beta}\right)=1\right\}=h\right\}$. Hence:

$$
\begin{aligned}
\text { card } & \left\{\xi \text { injective cycle } \mid \sum_{j=1}^{k} \operatorname{card} R_{j}(\xi)=l\right\} \\
& \leq \operatorname{card}\left\{v \mid L(v)=\alpha, \operatorname{card}\left\{1 \leq \beta \leq \alpha \mid D\left(v_{\beta}\right)=1\right\}=h\right\} \\
& \leq \operatorname{card}\left\{v \mid L(v)=\alpha, \operatorname{card}\left\{1 \leq \beta \leq \alpha \mid D\left(v_{\beta}\right)=1\right\} \geq h\right\} \\
& \leq\left(\begin{array}{l}
\alpha \\
h
\end{array}\right) \alpha^{h} n^{\alpha-h}=\left(\begin{array}{c}
\alpha \\
l
\end{array}\right) \alpha^{\alpha-l} n^{l} . \square
\end{aligned}
$$

5. Asymptotic freeness in the case of Weyl groups. For any positive integer $n$, let $A_{n}$ be a closed subgroup of the circle (i.e., $A_{n}=\mathbb{T}$ or $A_{n}=Z / r_{n} Z$ for some $r_{n}$ ), and let $G_{n}$ be the semidirect product $A_{n}^{n} \times_{\alpha_{n}} S_{n}$, where the action $\alpha_{n}$ of $S_{n}$ on $A_{n}^{n}$ is

$$
\alpha_{n}(t)\left(z_{1}, \ldots, z_{n}\right)=\left(z_{t^{-1}(1)}, \ldots, z_{t^{-1}(n)}\right) .
$$

We can view $G_{n}$ as a subgroup of $U(n)$, by identifying $g=$ $\left(\left(z_{1}, \ldots, z_{n}\right), t\right) \in G_{n}$ with the matrix having the $(i, j)$ entry equal to $z_{i}$, if $t(j)=i$, and to 0 , if $t(j) \neq i$. We have

THEOREM. The assertions (i) and (ii) of 3.1 are true for the series $\left(G_{n}\right)_{n=1}^{\infty}$.

To see this, we only need to take $\pi_{n}: G_{n} \rightarrow S_{n}$ the projection, and make the obvious remark that the inequality (12) appearing in the next lemma is valid:

LemMA. For $n \geq 1$, let $G_{n}$ and $H_{n}$ be closed subgroups of $U(n)$, and let $\pi_{n}$ be a continuous homomorphism of $G_{n}$ onto $H_{n}$, with the property that

$$
\operatorname{Tr} \pi_{n}(g) \geq|\operatorname{Tr} g|, \quad \text { for any } g \text { in } G_{n} .
$$

In this situation, if (i) and (ii) of 3.1 are valid for the series $\left(H_{n}\right)_{n=1}^{\infty}$, then they are also valid for the series $\left(G_{n}\right)_{n=1}^{\infty}$. 
Proof. Consider, for every $n \geq 1$, a standard-independent family $\left(f_{n, \omega}\right)_{\omega \in \Omega}$ of random unitaries in $G_{n}$.

Note first that $\left(\pi_{n} \circ f_{n}, \omega\right)_{\omega \in \Omega}$ is standard-independent in $H_{n}$. Indeed, for any $\omega_{1}, \ldots, \omega_{m}$ in $\Omega$ such that $\omega_{i} \neq \omega_{j}$ when $i \neq j$, and for any $\varphi_{1}, \ldots, \varphi_{m}$ in $C\left(H_{n}\right)$ :

$$
\begin{aligned}
& \int_{X} \varphi_{1}\left(\left(\pi_{n} \circ f_{n, \omega_{1}}\right)(x)\right) \cdots \varphi_{m}\left(\left(\pi_{n} \circ f_{n, \omega_{m}}\right)(x)\right) d P(x) \\
& =\int_{X}\left(\varphi_{1} \circ \pi_{n}\right)\left(f_{n, \omega_{1}}(x)\right) \cdots\left(\varphi_{m} \circ \pi_{n}\right)\left(f_{n, \omega_{m}}(x)\right) d P(x) \\
& \text { (2) } \stackrel{\text { of }}{=}{ }^{2.7} \prod_{q=1}^{m} \int_{G_{n}}\left(\varphi_{q} \circ \pi_{n}\right)(g) d g \\
& =\prod_{q=1}^{m} \int_{H_{n}} \varphi_{q}(h) d h .
\end{aligned}
$$

On the other hand, for any $\omega_{1} \neq \omega_{2} \neq \cdots \neq \omega_{m}$ in $\Omega$ and $\alpha_{1}, \ldots, \alpha_{m}$ in $\mathbb{Z} \backslash\{0\}$ we have

$$
\begin{aligned}
\mid \tau_{n}( & \left.f_{n, \omega_{1}}^{\alpha_{1}} \cdots f_{n, \omega_{m}}^{\alpha_{m}}\right) \mid \\
& \leq \frac{1}{n} \int_{X}\left|\operatorname{Tr}\left(\left(f_{n, \omega_{1}}^{\alpha_{1}} \cdots f_{n, \omega_{m}}^{\alpha_{m}}\right)(x)\right)\right| d P(x) \\
& \stackrel{(12)}{\leq} \frac{1}{n} \int_{X} \operatorname{Tr}\left(\left(\pi_{n} \circ\left(f_{n, \omega_{1}}^{\alpha_{1}} \cdots f_{n, \omega_{m}}^{\alpha_{m}}\right)\right)(x)\right) d P(x) \\
& =\frac{1}{n} \int_{X} \operatorname{Tr}\left(\left(\left(\pi_{n} \circ f_{n, \omega_{1}}\right)^{\alpha_{1}} \cdots\left(\pi_{n} \circ f_{n, \omega_{m}}\right)^{\alpha_{m}}\right)(x)\right) d P(x) \\
& =\tau_{n}\left(\left(\pi_{n} \circ f_{n, \omega_{1}}\right)^{\alpha_{1}} \cdots\left(\pi_{n} \circ f_{n, \omega_{m}}\right)^{\alpha_{m}}\right) .
\end{aligned}
$$

Taking into account the considerations of 3.2 , the last inequalities clearly finish the proof.

\section{REFERENCES}

[1] D. Voiculescu, Limit laws for random matrices and free products, Invent. Math., 104 (1991), 201-220.

[2] _ Free non-commutative random variables, random matrices and the $\mathrm{II}_{1}$ factors of free groups, in Quantum Probability and Related Topics (L. Accardi, editor), 1991, pp. 473-487.

Received April 30, 1991. 


\title{
PACIFIC JOURNAL OF MATHEMATICS
}

\author{
Founded by
}

E. F. BECKENBACH (1906-1982) F. WoLF (1904-1989)

\section{EDITORS}

$\begin{array}{ll}\begin{array}{l}\text { V. S. VARADARAJAN } \\ \text { (Managing Editor) }\end{array} & \begin{array}{l}\text { NiCHOLAS ERCOLANI } \\ \text { University of Arizona }\end{array} \\ \text { University of California } & \text { Tucson, AZ 85721 } \\ \text { Los Angeles, CA 90024-1555 } & \text { ercolani@math.arizona.edu } \\ \text { vsv@math.ucla.edu } & \text { R. FinN } \\ \text { HERBERT CLEMENS } & \text { Stanford University } \\ \text { University of Utah } & \text { Stanford, CA 94305 } \\ \text { Salt Lake City, UT 84112 } & \text { finn@gauss.stanford.edu } \\ \text { clemens@math.utah.edu } & \text { VAUGHAN F. R. JoNEs } \\ \text { F. MichAEL CHRIsT } & \text { University of California } \\ \text { University of California } & \text { Berkeley, CA 94720 } \\ \text { Los Angeles, CA 90024-1555 } & \text { vfr@math.berkeley.edu } \\ \text { christ@math.ucla.edu } & \text { STEVEN KERCKHoFF } \\ \text { THomas ENRIGHT } & \text { Stanford University } \\ \text { University of California, San Diego } & \text { Stanford, CA 94305 } \\ \text { La Jolla, CA 92093 } & \text { spk@gauss.stanford.edu } \\ \text { tenright@ucsd.edu } & \end{array}$

\author{
C. C. MOORE \\ University of California \\ Berkeley, CA 94720
}

MaRTin ScharlemanN

University of California

Santa Barbara, CA 93106

mgscharl@henri.ucsb.edu

\author{
HAROLD STARK \\ University of California, San Diego \\ La Jolla, CA 92093
}

\begin{tabular}{ll}
\multicolumn{1}{c}{ SUPPORTING } & INSTITUTIONS \\
UNIVERSITY OF ARIZONA & UNIVERSITY OF OREGON \\
UNIVERSITY OF BRITISH COLUMBIA & UNIVERSITY OF SOUTHERN CALIFORNIA \\
CALIFORNIA INSTITUTE OF TECHNOLOGY & STANFORD UNIVERSITY \\
UNIVERSITY OF CALIFORNIA & UNIVERSITY OF HAWAII \\
MONTANA STATE UNIVERSITY & UNIVERSITY OF TOKYO \\
UNIVERSITY OF NEVADA, RENO & UNIVERSITY OF UTAH \\
NEW MEXICO STATE UNIVERSITY & WASHINGTON STATE UNIVERSITY \\
OREGON STATE UNIVERSITY & UNIVERSITY OF WASHINGTON
\end{tabular}

The Supporting Institutions listed above contribute to the cost of publication of this Journal, but they are not owners or publishers and have no responsibility for its content or policies.

Mathematical papers intended for publication in the Pacific Journal of Mathematics should be in typed form or offset-reproduced (not dittoed), double spaced with large margins. Please do not use built up fractions in the text of the manuscript. However, you may use them in the displayed equations. Underline Greek letters in red, German in green, and script in blue. The first paragraph must be capable of being used separately as a synopsis of the entire paper. In particular it should contain no bibliographic references. Please propose a heading for the odd numbered pages of less than 35 characters. Manuscripts, in triplicate, may be sent to any one of the editors. Please classify according to the 1991 Mathematics Subject Classification scheme which can be found in the December index volumes of Mathematical Reviews. Supply name and address of author to whom proofs should be sent. All other communications should be addressed to the managing editor, or Julie Speckart, University of California, Los Angeles, California 90024-1555.

There are page-charges associated with articles appearing in the Pacific Journal of Mathematics. These charges are expected to be paid by the author's University, Government Agency or Company. If the author or authors do not have access to such Institutional support these charges are waived. Single authors will receive 50 free reprints; joint authors will receive a total of 100 free reprints. Additional copies may be obtained at cost in multiples of 50 .

The Pacific Journal of Mathematics (ISSN 0030-8730) is published monthly except for July and August. Regular subscription rate: $\$ 190.00$ a year (10 issues). Special rate: $\$ 95.00$ a year to individual members of supporting institutions.

Subscriptions, orders for numbers issued in the last three calendar years, and changes of address should be sent to Pacific Journal of Mathematics, P.O. Box 969, Carmel Valley, CA 93924, U.S.A. Old back numbers obtainable from Kraus Periodicals Co., Route 100, Millwood, NY 10546.

The Pacific Journal of Mathematics at P.O. Box 969, Carmel Valley, CA 93924 (ISSN 0030-8730) is published monthly except for July and August. Second-class postage paid at Carmel Valley, California 93924, and additional mailing offices. Postmaster: send address changes to Pacific Journal of Mathematics, P.O. Box 969, Carmel Valley, CA 93924.

PUBLISHED BY PACIFIC JOURNAL OF MATHEMATICS, A NON-PROFIT CORPORATION Copyright $(\mathcal{1} 1993$ by Pacific Journal of Mathematics 


\section{PACIFIC JOURNAL OF MATHEMATICS}

Volume $157 \quad$ No. $2 \quad$ February 1993

Strong integral summability and the Stone-Čech compactification of the half-line

JEFF CONNOR and MARY ANNE SWARDSON

The endlich Baer splitting property

225

THEODORE GERARD FATICONI

The formal group of the Jacobian of an algebraic curve

MARGARET N. FREIJE

Concordances of metrics of positive scalar curvature

PAWEL GAJER

Explicit construction of certain split extensions of number fields and constructing cyclic classfields

STANLEY JOSEPH GURAK

Asymptotically free families of random unitaries in symmetric groups

ALEXANDRU MiHAi NiCA

On purifiable subgroups and the intersection problem

TAKASHI OKUYAMA

On the incidence cycles of a curve: some geometric interpretations

LUCIANA RAMELLA

On some explicit formulas in the theory of Weil representation

R. RANGA RAO

An analytic family of uniformly bounded representations of a free product of 373 discrete groups

JANUSZ WYSOCZAŃSKI

Errata: "Dentability, trees, and Dunford-Pettis operators on $L_{1}$ "

MARIA GIRARDI and ZHIBAO HU

Errata: "Poincaré cobordism exact sequences and characterisation" 